\title{
Os peroneum imaging: normal appearance and pathological findings
}

\author{
Stefano Bianchi ${ }^{1} \cdot$ Chandra Bortolotto $^{2} \cdot$ Ferdinando Draghi $^{2}$
}

Received: 29 October 2016 /Revised: 11 December 2016 / Accepted: 19 December 2016/Published online: 5 January 2017

(C) The Author(s) 2017. This article is published with open access at Springerlink.com

\begin{abstract}
The os peroneum (OP) is a small sesamoid bone located inside the peroneus longus tendon (PLT), close to the cuboid. The OP can be the cause of pain and can be associated with lesions of the PLT. OP involvement in PLT disorders is frequently misdiagnosed by radiologists. Painful os peroneum syndrome (POPS) refers to a variety of conditions presenting with pain localized on the lateral aspect of the cuboid area. The syndrome can be observed as a consequence of local acute trauma such as ankle sprains or chronic overuse. Because of its intra-tendinous location, in tears of the peroneus longus tendon, the OP can show changes in its morphology or position, depending on the location of the tendon's tear. Based on the level of the PLT tears, we propose a classification in three subtypes: tears localized proximal to the os peroneum (type I), at its level (type II) or distal to it (type III). These tears present with different changes on OP morphology or location. The aim of this article is to review the normal anatomy, imaging appearance and differential diagnosis of disorders of the OP as well as post-treatment imaging.

\section{Teaching points}

- PLT tears can be classified in three subtypes according to OP location.

- POPS is characterized by pain on the lateral aspect of the cuboid.

- OP involvement in PLT disorders is frequently misdiagnosed by radiologists.
\end{abstract}

Chandra Bortolotto

chandra.bortolotto@gmail.com

CIM SA, Cabinet Imagerie Médicale, Genève, Suisse

2 Radiology Institute, University of Pavia, Pavia, Italy
Keywords Os peroneum · Peroneus longus tendon · Painful os peroneum Syndrome $\cdot$ Ankle injuries $\cdot$ Diagnostic imaging

\section{Introduction}

Disorders of the peroneal tendons (PT) are frequent in the everyday practice of rheumatologists, orthopaedics, sports medicine doctors and physiotherapists [1-4]. The os peroneum (OP) is a small sesamoid bone located inside the PLT, close to the cuboid [5]. It is seen in 5-26\% of the population [6] and is bilateral in $60 \%$ of patients [7]. OP involvement in PT pathologies is frequently misdiagnosed by radiologists $[8,9]$. Nevertheless, the OP can be the cause of pain and can be associated with lesions of the PLT [10]. While in the majority of cases history and physical examination are able to guide towards a correct diagnosis, imaging is often necessary to confirm the clinical suspicion and choose between surgical and medical treatment. The aim of this article is to review the normal anatomy and imaging appearance of disorders of the OP.

\section{Normal anatomy of the OP (Fig. 1)}

The PT complex is made up of the muscles and tendons of the PLT and peroneus brevis tendon (PBT), their common synovial sheath, the superior and inferior retinaculum, and the OP $[5,11-15]$.

The peroneal longus (PLM) and brevis (PBM) muscles are located inside the external compartment of the leg, lateral to the fibula. The PLM originates from the proximal portion of the lateral surface of the fibula and from the nearby intermuscular septum. The PBM originates from the distal 


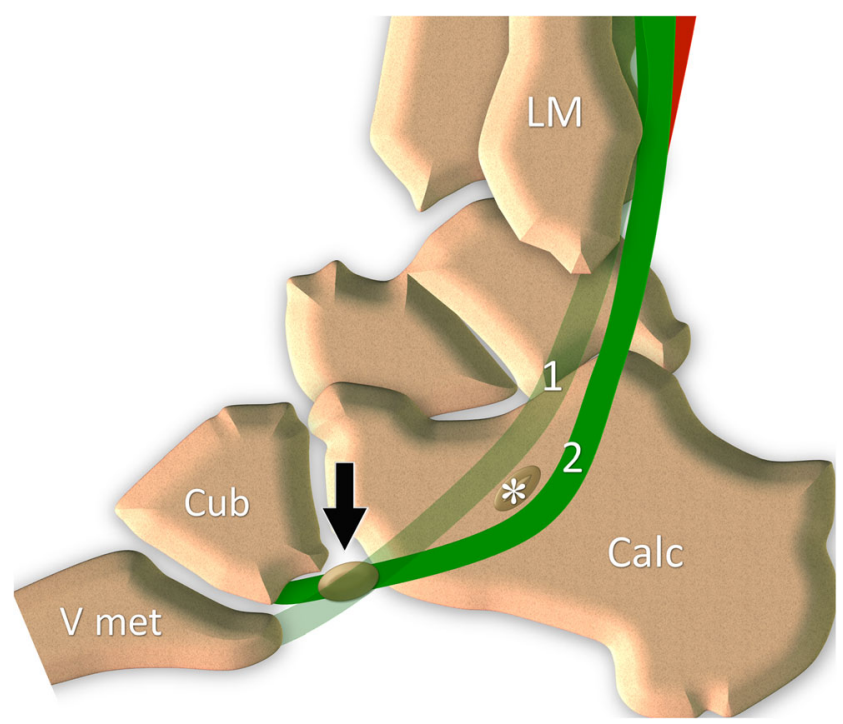

Fig. 1 Os peroneum normal anatomy. Peroneal tendons (peroneus brevis 1 , peroneus longus 2) travel along the lateral surface of the calcaneus that presents a small bony prominence, the peroneal tubercle (asterisk). The $\mathrm{OP}$ can be found inside the tendon at the level of the calcaneocuboid joint (black arrow). LM lateral malleolus, Calc calcaneus, Cub cuboid, $V$ met fifth metacarpal bone

third of the lateral surface of the fibula and from the nearby intermuscular septum. At the level of the leg's median third, the PLM extends through a superficial, flat aponeurosis that covers the PBM. The PBM presents a myoaponeurotic junction distally located with regard to that of the PLM. Both muscles are innervated by the peroneal superficial nerve. The peroneal muscles are supplied by two principal source arteries, the anterior tibial artery and the peroneal artery.

At the malleolar region, the two tendons reflect against the posterior aspect of the lateral malleolus. At this level they run inside an osteofibrous tunnel (proximal tunnel) made up of the concave bony gutter and the thick fibrous superior retinaculum. This inserts into the lateral surface of the malleolus and guarantees the stability of the tendons during ankle movement, thus preventing their anterior luxation during movement of the ankle.

Distal to the lateral malleolus, the PT travel along the lateral surface of the calcaneus, which presents a small bony prominence, the peroneal tubercle (PTub). The peroneus brevis tendon (PBT) runs superiorly while the PLT runs inferiorly to it. At this level, the two tendons run inside another osteofibrous tunnel (distal tunnel), stabilized against the calcaneus thanks to the inferior retinaculum, which inserts on the apex of the PTub. The tubercle acts as a pulley for the PLT that reflects on its inferior surface.

Distal to the PTub, the PBT travels straight forward, to insert into the base of the fifth metatarsal bone.

The PLT, after reflecting against the cuboid, reaches the plantar region and attaches to the first and second metatarsal bones.
The two PT are enclosed by a common synovial sheath allowing optimal sliding of the tendons during movements of the ankle and/or contraction of the peroneal muscles. The sheath is made of a parietal layer adherent to the paratendinous soft tissue and a visceral layer adherent to the tendons. Proximal to the PTub the sheath divides to individually surround the two tendons. In some cases, the sheath surrounding the PLT is longer and extends from the cuboid tunnel to the plantar aspect of the foot. In physiologic conditions, it contains only a small amount of synovial fluid.

The OP is a small sesamoid bone which can be found inside the PLT at the level of the calcaneocuboid joint. The OP is ossified in around $20 \%$ of the population, and it is bilateral in around $60 \%$ of cases [7, 16, 17]. This small sesamoid bone can show different sizes and can also be bipartite or multipartite. A recent cadaveric study described the normal shape and size of the OP on 36 cadavers [18]. The ossicle presented a flattened oval shape, with one or two concave articular deep surfaces. The average thickness was $4 \mathrm{~mm}$ and length $13 \mathrm{~mm}$. A bipartite bone is common and can be seen in around $30 \%$ of cases [19].

The presence of an OP or a bipartite OP can be clinically significant. The differential diagnosis between the ossicle and a cortical avulsion or a soft tissue calcification can be difficult in patients with pain in the lateral side of the foot. Similarly, a multipartite bone must be differentiated from a fractured one.

The origin of OP development is controversial. Some authors advocate an embryonic development of the ossicle [20], while others [18] believe that the OP develops from a "stress response". A recent cadaveric study from Guimera and colleagues demonstrates that a precursor of the OP is already present during the foetal period [20]. Mittal et al. propose that development of the OP follows local stresses to the tendon, leading to its thickening and secondary ossification [18].

\section{Normal imaging of the OP}

The OP can be visualized with several imaging techniques.

Internal oblique radiographs of the foot can visualize the OP as an oval, well-corticated ossicle located close to the calcaneocuboid joint. The size, margins and location of the OP are easily assessed (Fig. 2A). In multipartite OP, two or more oval fragments showing well-defined sclerotic margins are noted (Figs. $2 \mathrm{~b}$ and 3 ). Accurate evaluation of the bone margins is of the utmost importance for differential diagnosis with a fracture.

Ultrasound (US) allows an analysis of the OP when using a high-frequency transducer and a rigorous examination technique (Fig. 4) [5, 21]. The examination starts at the PTub, which can be visualized in the oblique coronal plane as a hyperechoic eminence. Once the PTub is detected, the transducer is moved forward along the PLT to the cuboid. When 


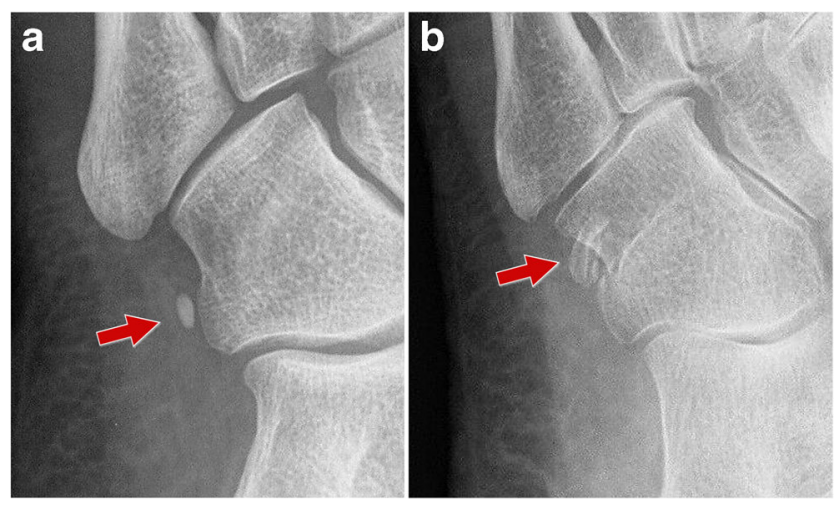

Fig. 2 Normal os peroneum - standard radiograph. 2A Single os peroneum (arrow) visualized as an oval, well-corticated ossicle located close to the calcaneocuboid joint. $2 B$ Bipartite os peroneum (arrow): two or more round fragments showing well-defined sclerotic margins

present, the OP is visualized at this level as a hyperechoic structure showing posterior shadowing [22]. Once detected, the OP must be analysed on both axial and coronal oblique planes. Although US allows evaluation of the presence, multipartite appearance and size of the sesamoid, only the outer surface of the ossicle can be judged. The distal portion of the PLT can then be assessed in the plantar aspect of the foot. US is of value in guiding local interventional procedures such as corticosteroid injections in the peroneal tendon sheath, calcaneocuboid joint and local soft tissues [23].

Computed tomography (CT) allows a more precise evaluation of the OP, particularly when it is realized on a

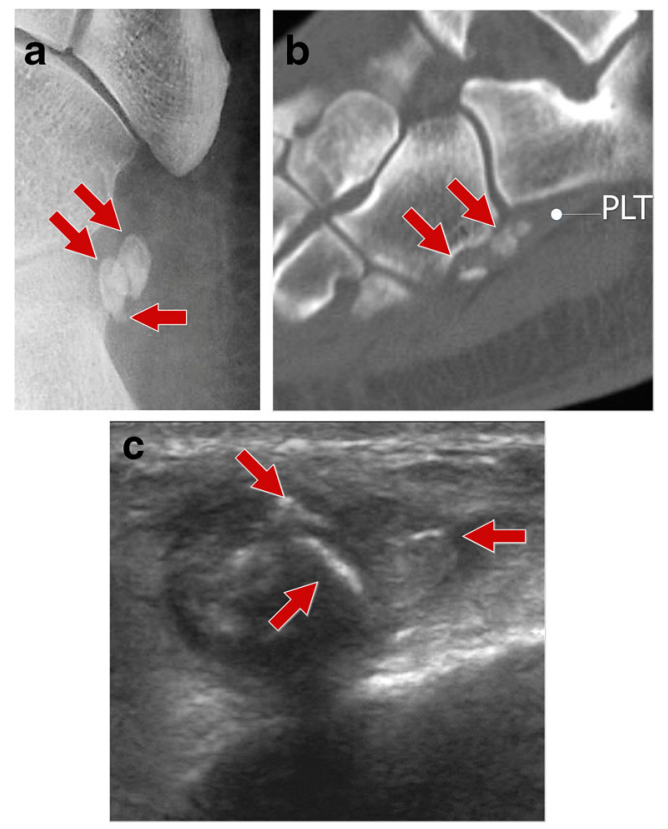

Fig. 3 Multipartite os peroneum (arrows; different patients). $3 A$ Standard radiograph. $3 B-C$ Computed tomography and ultrasound. Accurate evaluation of the bone margins is of the utmost importance for differential diagnosis with a fracture. $\mathrm{CT}$ is the technique of choice to differentiate a multipartite OP from a non-displaced fracture. PLT peroneus longus tendon

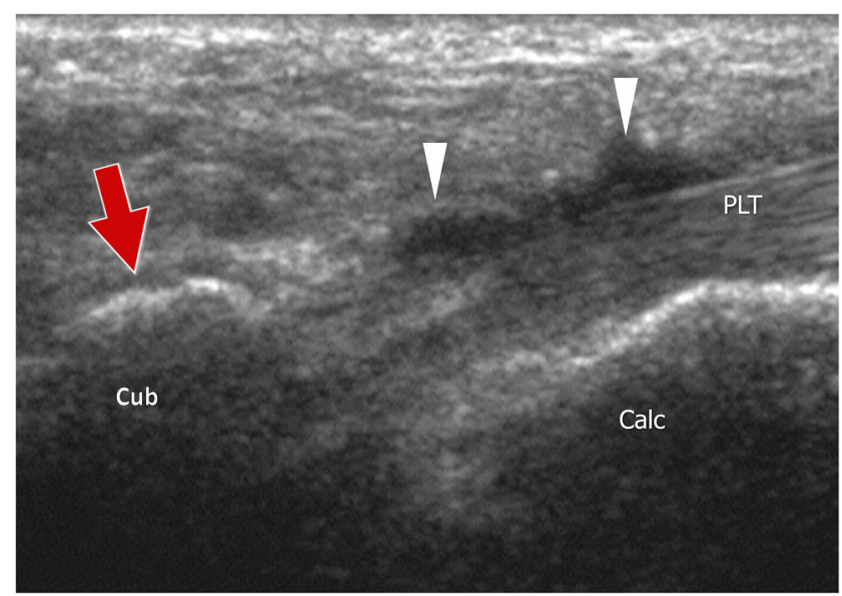

Fig. 4 Normal os peroneum - ultrasound. The transducer is moved forward along the PLT until the cuboid $(c u b)$. A small amount of synovial fluid can be visualized along the PLT (arrowhead). When present, the OP is visualized at this level as a hyperechoic structure showing posterior shadowing (arrow)

multidetector scanner with sub-millimetre collimation and oblique reconstruction on several planes (Fig. 5). It is the technique of choice for differentiating a multipartite OP from a non-displaced fracture.

Magnetic resonance imaging (MRI) allows an accurate evaluation of the OP (Fig. 6). The OP can be appreciated on axial, sagittal and coronal planes, especially if a $3 \mathrm{D}$ technique and small slice thickness are employed. Since the OP is located in a transition zone between the lateral and plantar course of the peroneus longus tendon, it is important to properly study this structure by orienting axial planes perpendicular to the long axis of the metatarsal shaft [22]. A correct orientation of planes helps minimize "magic angle" artefacts and also to

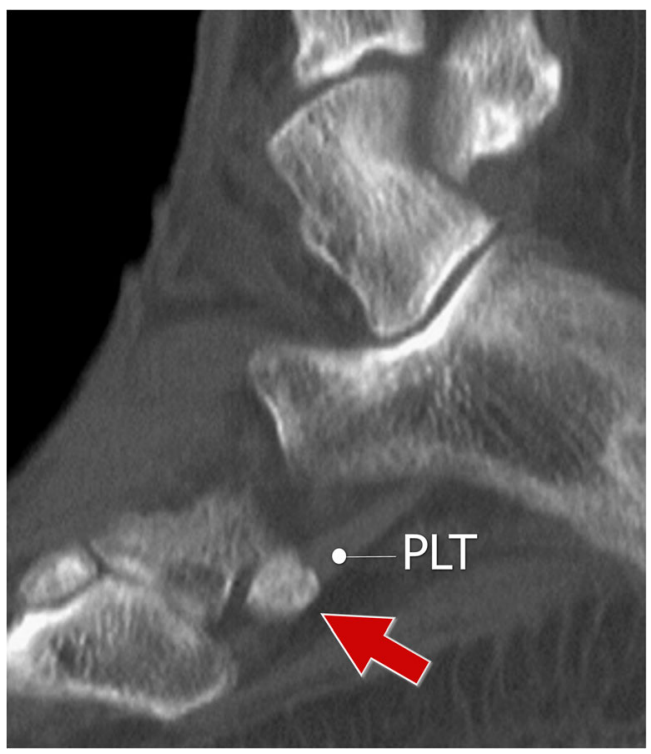

Fig. 5 Normal os peroneum - computed tomography. Sagittal reconstruction along the PLT allows a precise evaluation of the OP (arrow) 


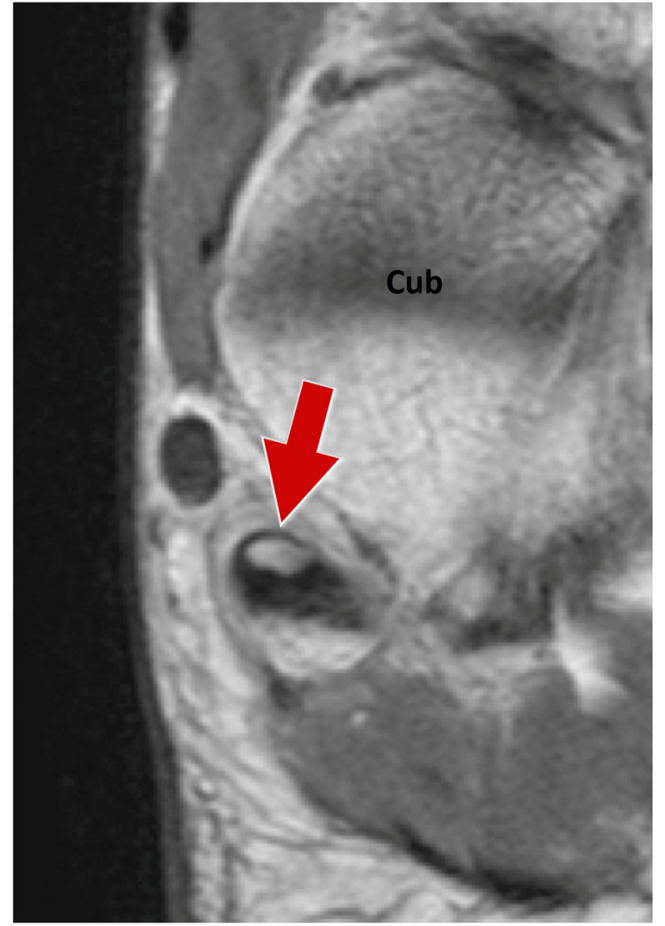

Fig. 6 Normal os peroneum - magnetic resonance imaging. Coronal T1w plane. MRI allows optimal evaluation of the OP (arrow) bone marrow, which shows a signal similar to that of the adjacent cuboid and also correctly depicts pathologies of the synovial sheaths. $\mathrm{Cub}$ cuboid

correctly depict pathologies of the synovial sheaths. MRI allows optimal evaluation of the OP bone marrow, which shows a signal similar to that of the adjacent cuboid, hyperintense on $\mathrm{T} 1 \mathrm{w}$ sequence and hypointense on $\mathrm{T} 2 \mathrm{w}$ with fat suppression. Assessment of the cortex is difficult because of the hypointense signal of both the cortical bone and the tendon which encases it.

When no proper OP is visible, and only a fibrocartilaginous node is present, it is important to avoid one of the most frequent pitfalls of this situation. On MRI, the fibrocartilaginous node appears as a "pseudo tear". Due to its chemical composition, it has an intermediate signal and can be mistaken as a real tear by an inexperienced radiologist [24].

Bone scintigraphy can detect the uptake in the area of the OP in chronic lateral foot pain with an unclear clinical history (micro-traumas or mismanaged non-recent traumas). In these cases, the nuclear medicine specialist must bear in mind the possibility that an uptake in this area can be related to a pathology of the OP [25].

\section{Imaging findings in pathologic conditions}

\section{Painful os peroneum syndrome (POPS)}

POPS refers to a variety of conditions presenting with pain localized on the lateral aspect of the cuboid area or on the OP [10].
The syndrome can be observed as a consequence of local acute trauma such as ankle sprains. In these cases, tears of the PLT or acute OP fractures are typically observed. Chronic overuse can also lead to POPS secondary to bone marrow oedema of the OP and cuboid, OP stress fractures, local impingement or partial tears of the PLT $[10,17,26,27]$. As a consequence of the marked reflection of the PLT against the cuboid and secondary local stresses, it is not surprising that local friction between the two structures can lead to a local mechanical conflict [17, 28, 29]. Anatomic variation such as a hypertrophic OP, as well as overuse including cases of sport activities or hyper-supination of the ankle, can also facilitate a local impingement (Fig. 7) [10]. Patients present with well-localized pain over the lateral aspect of the tarsal region, usually exacerbated by walking, running and other physical activities.

In acute fractures, standard radiographs show multiple fragments of the OP, typically presenting sharp borders. This must be differentiated from a multipartite OP, in which the fragments are rounded and present regular and sclerotic cortical edges [30]. Correlation with clinical findings can aid in differential diagnosis. In chronic stresses, radiographs can show an enlarged and sclerotic OP suggestive of a stress fracture (Fig. 8).

In the majority of cases, US does not show abnormalities of the OP [28], which shows a regular and smooth cortical margin (Fig. 7b). Bone marrow oedema cannot be detected by US. Colour Doppler US can detect inflammatory hyperaemia of the adjacent soft tissues reflecting a local inflammation. These findings are detected only in the most severe cases and need an adequate examination technique as well as a high degree of suspicion by the examiner. When needed, US allows a realtime, accurate local therapeutic injection.
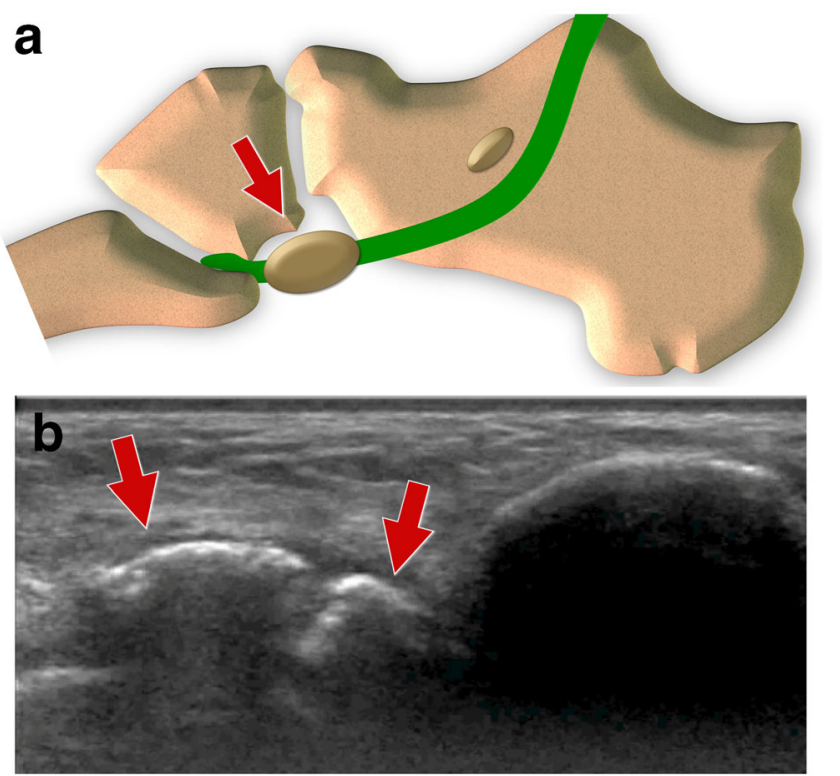

Fig. 7 Hypertrophic os peroneum. $7 A$ Scheme $7 B$ Ultrasound. Hypertrophic OP facilitated a local impingement 


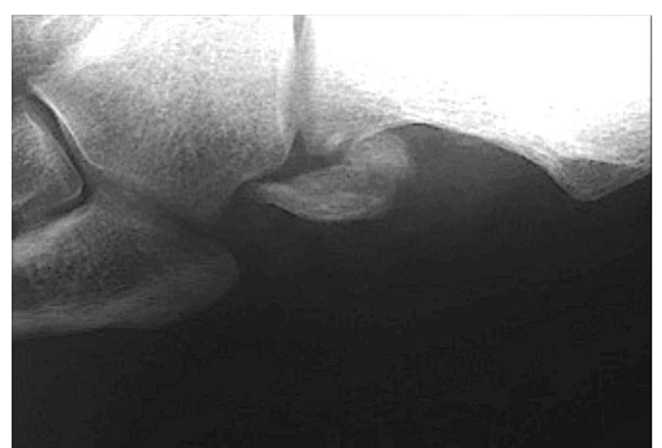

Fig. 8 POPS - standard radiograph. Radiographs can show an enlarged and sclerotic OP suggestive of a stress fracture

CT can assess the morphology of the OP well and allows a definite diagnosis of its acute or stress fractures, and in some cases points out erosions of the cuboid cortical bone [28]. This technique does not allow detection of bone marrow oedema or changes in the adjacent soft tissues.

MRI is the gold standard in diagnosing POPS, since it shows pathologic changes of the bone marrow of the OP and cuboid as well as of soft tissues. Oedema appears hypointense on T1w sequence and hyperintense on T2w sequence with fat suppression (Fig. 9). After gadolinium administration, a local enhancement is detected. Nevertheless, MRI is inferior to CT in the assessment of the cortex of the OP.

Bone scintigraphy can detect the uptake in the area of the OP in chronic lateral foot pain. In these cases, that uptake can be related to a pathology of the OP [25] (Fig. 10).

\section{OP in PLT tears}

Because of its intra-tendinous location, in tears of the PLT, the OP shows changes in its morphology or position. Based on a
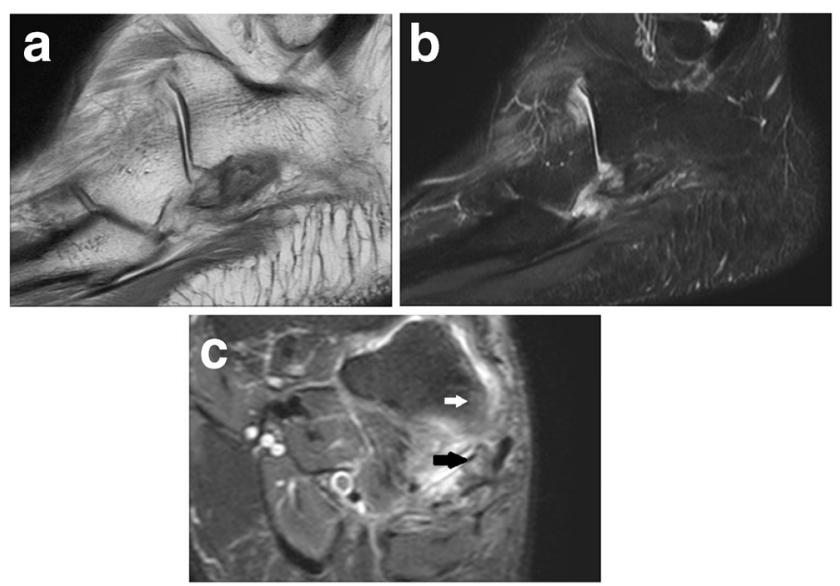

Fig. 9 POPS - magnetic resonance imaging. 9A T1w image, sagittal plane. $9 B \mathrm{~T} 2 \mathrm{w}$ fat suppression image, sagittal plane. $9 C \mathrm{~T} 2 \mathrm{w}$ fat suppression, coronal plane. MRI shows oedema of the bone marrow of the OP (black arrow) and cuboid (white arrow) as well as inflammatory changes in soft tissues
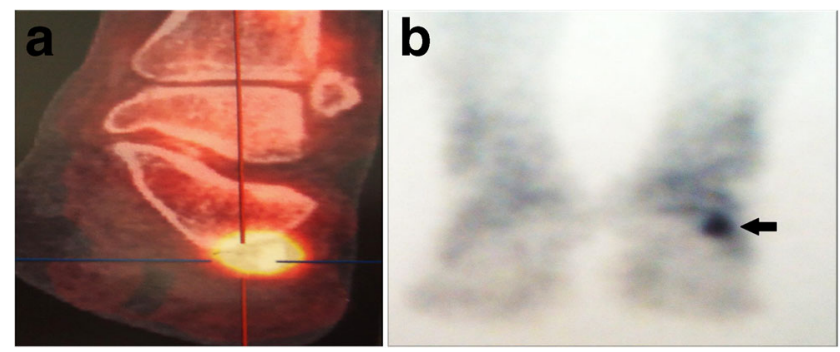

Fig. 10 POPS - nuclear medicine. 10A Positron emission tomography. $10 B$ Bone scintigraphy. Nuclear medicine can detect the uptake in the area of the OP (arrow) in chronic lateral foot pain

review of the literature $[1-4,8-10,15,16,23]$, as well as our practice experience, we propose a classification of tears of the PLT into three subtypes: tears localized proximal to the OP (type I), at its level (type II) or distal to it (type III) (Fig. 11).

\section{Type I tears (Fig. 12)}

When the PLT tear is proximal to the OP, the ossicle presents a normal appearance and localization. This lesion cannot be diagnosed with radiographs or CT, which show a normal OP, but can be detected with ultrasound and MRI as irregularity of the tendons with local discontinuity usually associated with tenosynovitis.

\section{Type II tears (Fig. 13)}

Tears of the PLT at the OP level present as ossicle fractures with two or more bone fragments of different sizes. As noted, OP fractures can be acute or chronic and present as a POPS syndrome. Diabetes mellitus is a factor facilitating fractures of the OP [31]. Whatever the pathologic mechanism, it implies an acute mechanical tension exerted by the PLT. Due to the persistent mechanical tension exerted by the PLT, a
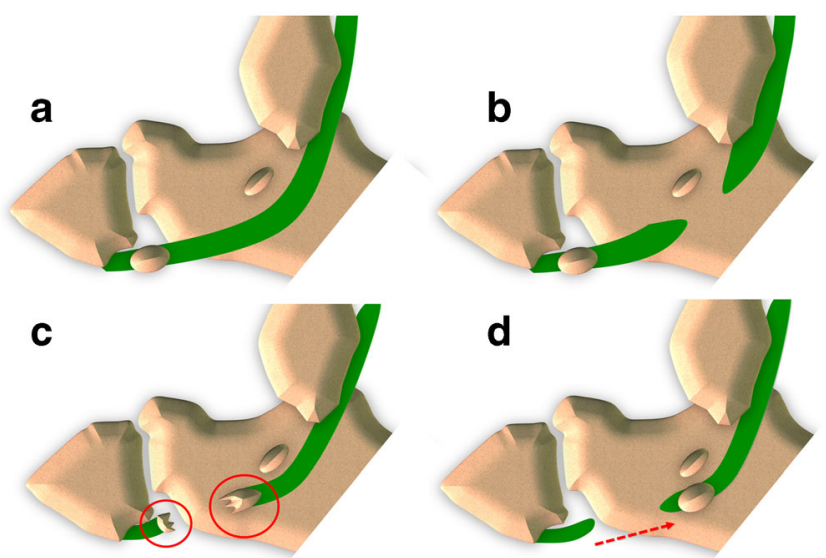

Fig. 11 PLT tears - Scheme. A normal, $B$ tear localized proximal to the OP (type I), $C$ tear localized at OP level (type II), $D$ tear localized distal to the OP (type III) 
Fig. 12 PLT tear type I. 12A Scheme $12 B$ Ultrasound image. Longitudinal scan (tendon long axis). Arrows show the two tendon stumps and the gap between them. $12 C$ Ultrasound image. Transverse scan (tendon short axis). The peroneus longus tendon is not visible and is replaced by effusion (arrow). Peroneal calcaneal ligament (arrowhead). PBT peroneus brevis tendon. 12D Magnetic resonance imaging. Retracted proximal stump of the peroneus longus tendon (arrow)
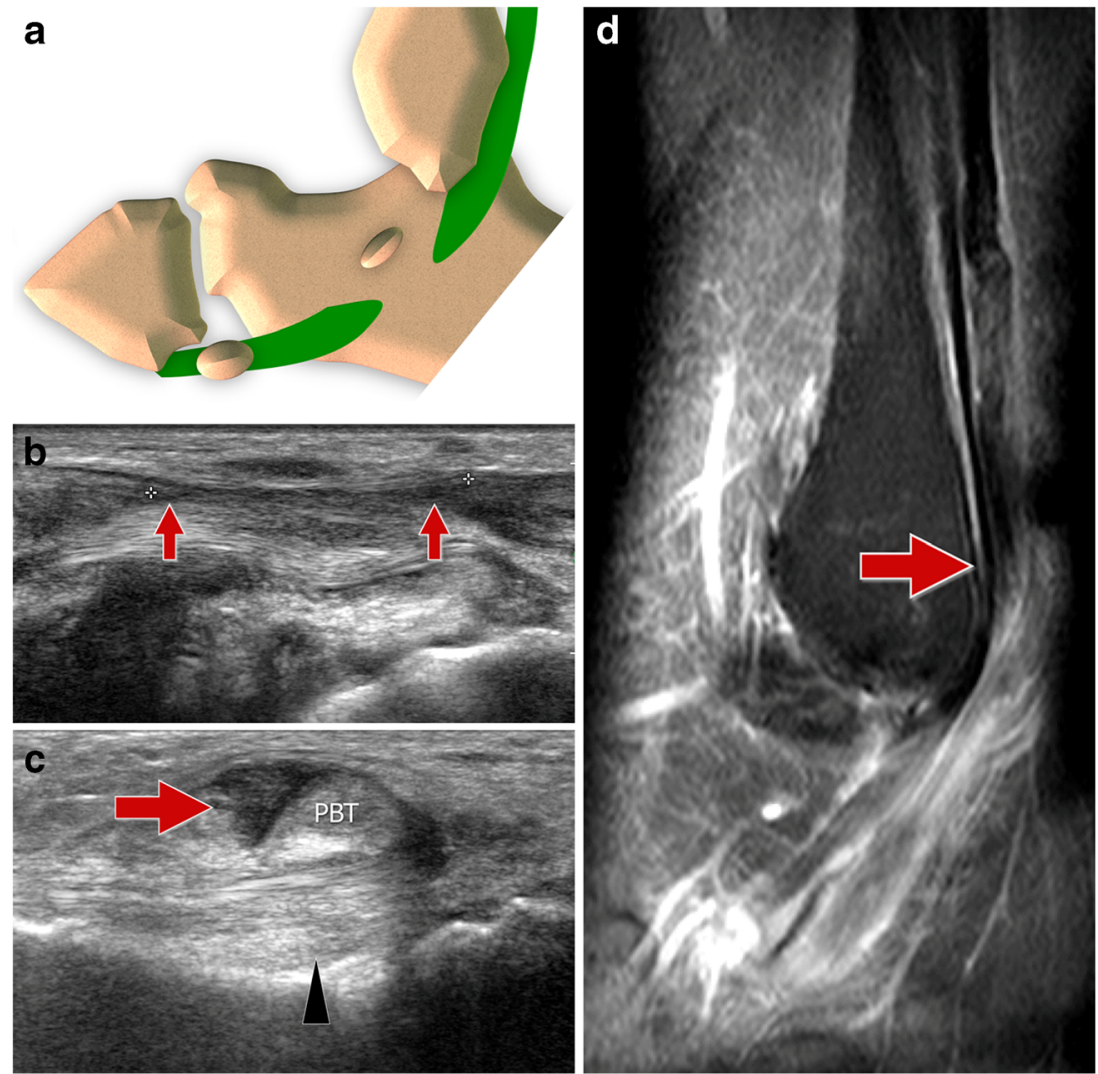

displacement of the fracture fragments can be readily evident or visualized only in the following days (delayed displacement).

Fig. 13 PLT tear type II. 13A

Scheme $13 B$ Standard internal oblique radiographs. On the left side, a lesion at the level of the OP with separation between fragments (arrows) of more than $6 \mathrm{~mm}$. On the right side, the contralateral normal OP (arrow). $13 C$ Ultrasound. US clearly shows the two fragments (arrows) and the hematoma between them (arrowhead)
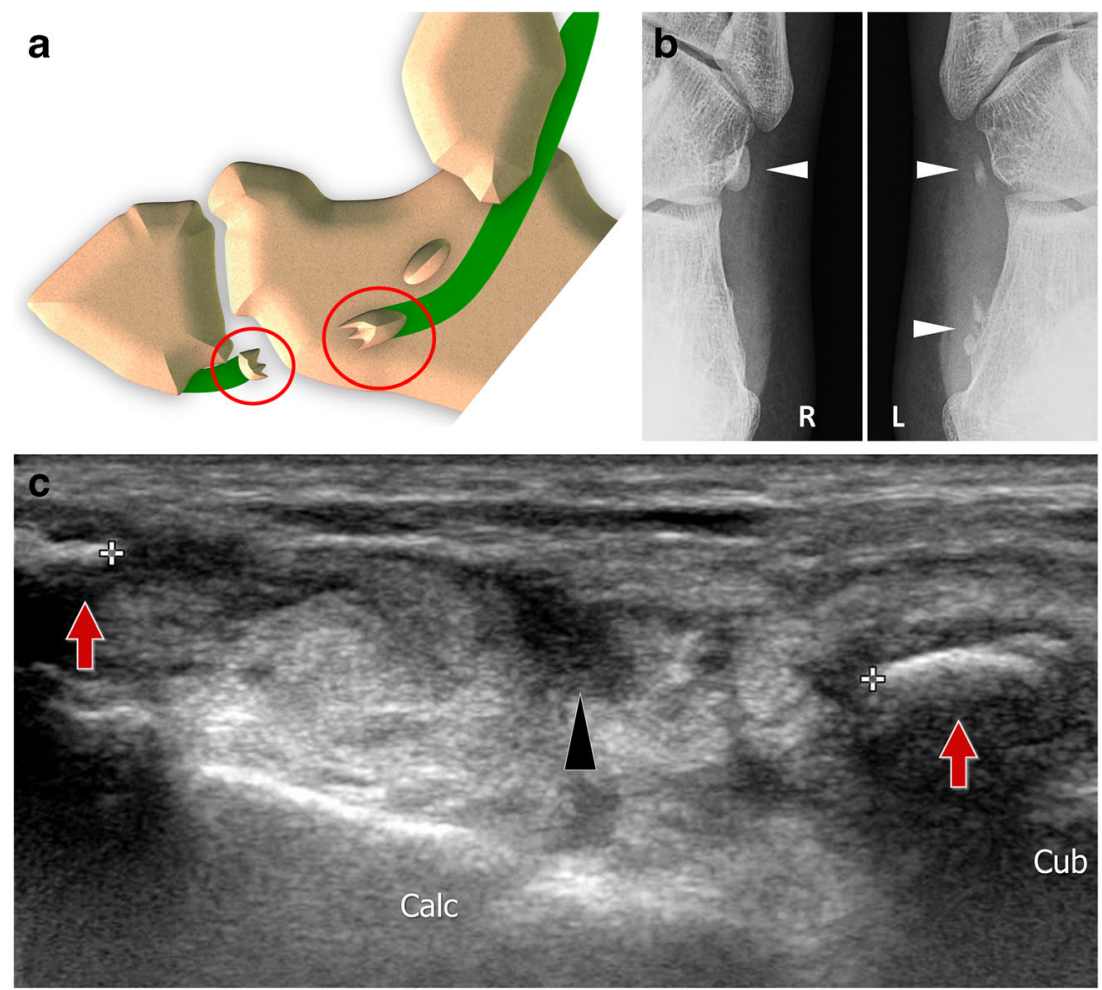
usually larger (Fig. 13b). A separation between fragments of more than $6 \mathrm{~mm}$ or significantly increased displacement in subsequent control radiographs is associated with a complete lesion of the PLT [32, 33]. A progressive displacement, with radiographs obtained both immediately after the trauma and some days later showing a progressive increase in fragment distance, is an argument in favour of a complete tendon lesion [19, 32-34]. The proximal fragment is usually found anterior to the PTub of the calcaneus, inside the inferior osteofibrous tunnel. After strong contraction, the proximal fragment can be found posteriorly to the PTub, at the level of the posterior subtalar joint. When the fracture is not displaced, its irregular appearance and the absence of round sharp margins are the main aspects to take into account for a differential diagnosis between a fracture and a multipartite OP. In patients with a posterior displacement which is not readily evident, obtaining a contralateral radiograph can easily show even slight migration of the ossicle.

US can easily show a fracture of the OP when a significant displacement $(>6 \mathrm{~mm})$ of the posterior fragment is present. In these cases, US shows the bone fragments localized inside the tendon along the lateral side of the calcaneus. The retracted proximal fragment is usually found at the level of the PTub. The distal fragment can be more difficult to detect because of its smaller size. The distance between the two can be accurately measured (Fig. 13c). This can aid preoperative planning in order to correctly plan surgical incision points. In recent traumas, US can also show the hematoma between the two fragments and the associated inflammatory soft tissue phenomena. Colour Doppler can detect local hyperaemia. Local pressure exerted under US guidance is painful.

CT can detect the presence of the fracture even if minimally displaced, especially if performed with a multidetector scanner. Retraction of the proximal fragment is easily appreciated.

MRI is less useful than CT in analysing the bone cortex in non-displaced fractures, but allows the detection of bone marrow oedema and adjacent soft tissue inflammation in fat saturation images. In displaced fractures, MRI can easily detect the position of the retracted fragment and can demonstrate possible impingement of the retracted fragment at the level of the PTub inside the distal tunnel. MRI impingement features are similar to those of classic POPS but located more proximally. We call this lesion the "proximal POPS". In addition, MRI shows tendon lesions and effusion inside the synovial sheath (seen as a hyper-signal on $\mathrm{T} 2 \mathrm{w}$ sequence with fat suppression).

\section{Type III tears (Fig. 14)}

When tears of the PLT are located distal to the OP, the sesamoid shows a normal appearance even though it is posteriorly displaced, because of the traction exerted by the peroneus longus
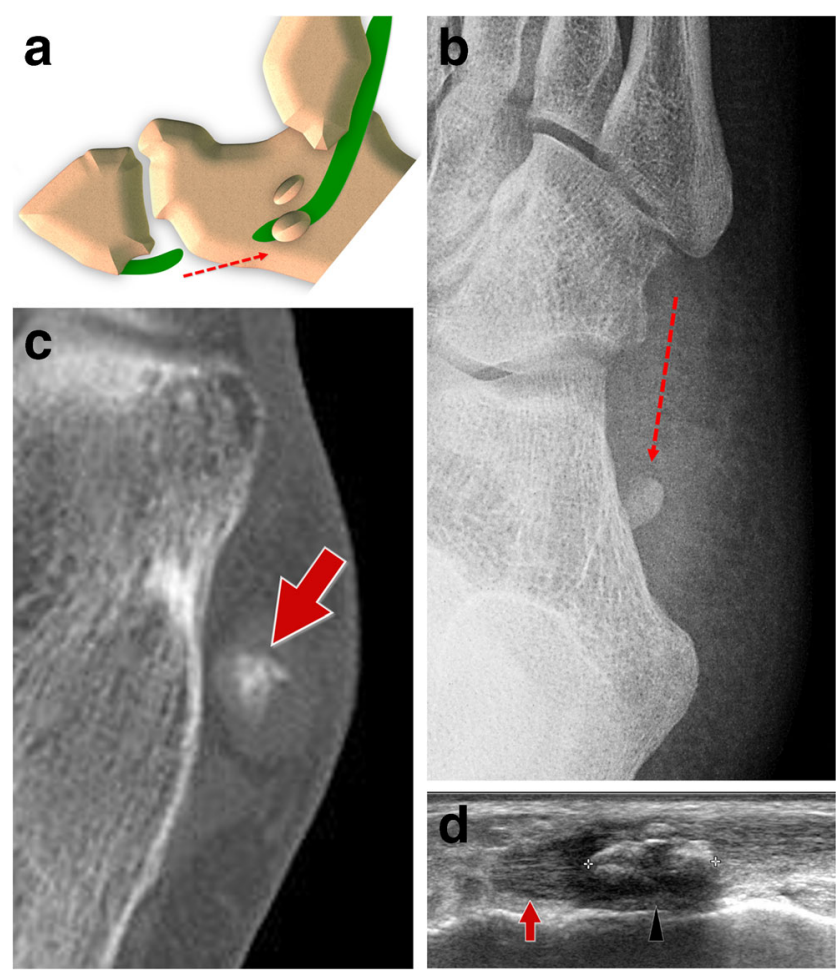

Fig. 14 PLT tear type III standard retraction. 14A Scheme $14 B$ Standard internal oblique radiograph. The OP shows a normal appearance but is posteriorly dislocated (generally less than $2 \mathrm{~cm}$ ). $14 C$ Computed tomography. CT shows a regular appearance of the displaced sesamoid bone. 14D Ultrasound. The degree of the dislocation and the size of the proximal tendinous stump (arrows) are easily measured. OP (arrowhead)

$[36,37]$. Displacement is generally less than $2 \mathrm{~cm}[38]$, as the sesamoid bone is stuck inside the distal osteofibrous tunnel at the level of the PTub. As already noted for type II tears, after strong contraction, the OP can be found posterior to the PTub, at the level of the posterior subtalar joint (Fig. 15). In this case, it can be misinterpreted as an os trigonum in an unusual position. This happens rarely, especially if the OP is small in size [33].

The internal oblique radiograph is the best way to highlight the posterior unusual localization of the OP. On lateral radiographs, this small bone is hard to localize because it is superimposed on the calcaneus. For these reasons, this diagnosis can be challenging for less experienced radiologists and is generally easier when a clinical suspicion is present. A comparison with previous radiographs is extremely important because it can confirm the displacement of the OP (with a normal localization in the initial radiograph) $[19,34,39]$. When previous radiographs are not available, a radiograph of the contralateral ankle can be obtained in order to show a typical localization of the contralateral OP if one is present.

US easily confirms type 3 tears. The degree of the displacement and the size of the proximal tendinous stump are easily measured. This can aid preoperative planning in order to correctly plan surgical incision points. Lastly, the alterations of 
Fig. 15 PLT tear type III proximal retraction. $15 \mathrm{~A}$ Scheme: proximal retraction after strong contraction. $15 B-C$ Lateral and internal oblique radiograph. The OP (arrows) can be found at the level of the posterior subtalar joint. $15 D-E$ Magnetic resonance imaging and ultrasound. The $\mathrm{OP}$ (arrows) can be found posteriorly to the PTub
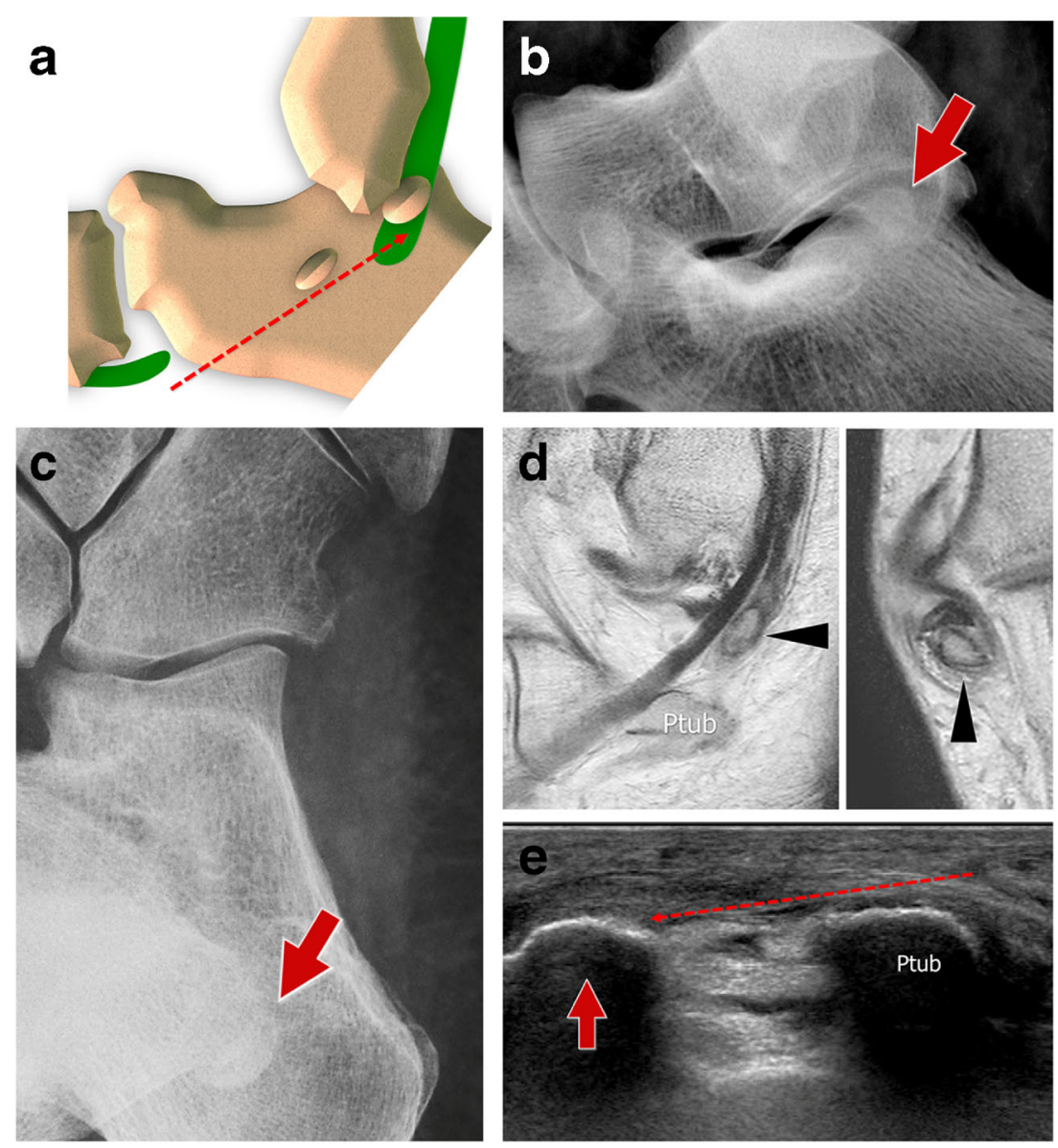

the tendon sheaths are visible with US: effusion, sheath thickening and hyperaemia.

CT shows a regular appearance of the displaced sesamoid bone. The main differential diagnosis is with an accessory os subfibulare. Unfortunately, the tendon lesion is not evident in most cases.

This type of lesion can be identified with MRI, especially after contrast medium injection. Coronal images easily show the proximal stump, the displaced OP and the empty tendon sheaths distally located.

\section{Post-treatment imaging findings}

Treatments for OP-related pathologies are extremely varied, ranging from non-operative treatments even in high-level athletes [40], to repair of the fractured ossicle or excision of both or just the proximal fragment in fractures [6]. Newer approaches include those based on tendoscopic intervention [41].

To our knowledge, no studies are available on postoperative imaging of the PLT and OP. Hypothetically, imaging can detect acute complications such as infection or re-rupture and late complications such as reflex sympathetic dystrophy syndrome (RSDS) or neuromas (e.g. sural nerve distal cutaneous branches travel on the lateral aspects of the ankle).

\section{Conclusions}

The actual prevalence of the OP pathology is difficult to assess, but in our opinion, it is probably underestimated. Pathologic involvement of OP is frequently misdiagnosed by radiologists.

When an OP fracture is suspected, standard radiographs allow for a correct diagnosis only if carefully evaluated. In case of doubt, the contralateral ankle or previous radiographs must be used for comparison.

MRI is an extremely powerful tool but needs proper plane and field of view selection. It is the gold standard for the detection of POPS.

When a PLT tear is suspected, US can be used as a first approach. Also for US, contralateral ankle evaluation is extremely useful. 


\section{Compliance with ethical standards}

Conflict of interest The authors declare that they have no conflict of interest.

Ethical approval All procedures performed in studies involving human participants were in accordance with the ethical standards of the institutional and/or national research committee and with the 1964 Helsinki declaration and its later amendments or comparable ethical standards.

Informed consent Informed consent was obtained from all individual participants included in the study.

Open Access This article is distributed under the terms of the Creative Commons Attribution 4.0 International License (http:// creativecommons.org/licenses/by/4.0/), which permits unrestricted use, distribution, and reproduction in any medium, provided you give appropriate credit to the original author(s) and the source, provide a link to the Creative Commons license, and indicate if changes were made.

\section{References}

1. Heckman DS, Gluck GS, Parekh SG (2009) Tendon disorders of the foot and ankle, part 1: peroneal tendon disorders. Am J Sports Med 37(3):614-625

2. Philbin TM, Landis GS, Smith B (2009) Peroneal tendon injuries. J Am Acad Orthop Surg 17(5):306-317

3. Selmani E, Gjata V, Gjika E (2006) Current concepts review: peroneal tendon disorders. Foot Ankle Int 27(3):221-228

4. Trevino S, Baumhauer JF (1992) Tendon injuries of the foot and ankle. Clin Sports Med 11(4):727-739

5. Bianchi S, Martinoli C, Gaignot C, De Gautard R, Meyer JM (2005) Ultrasound of the ankle: anatomy of the tendons, bursae, and ligaments. Semin Musculoskelet Radiol 9(3):243-259

6. Sammarco VJ, Cuttica DJ, Sammarco GJ (2010) Lasso stitch with peroneal retinaculoplasty for repair of fractured os peroneum: a report of two cases. Clin Orthop Relat Res 468(4):1012-1017

7. Peacock KC, Resnick EJ, Thoder JJ (1986) Fracture of the os peroneum with rupture of the peroneus longus tendon: a case report with review of the literature. Clin Orthop 202:223-226

8. Brandes CB, Smith RW (2000) Characterization of patients with primary peroneus longus tendinopathy: a review of twenty-two cases. Foot Ankle Int 21(6):462-468

9. Dombek MF, Lamm BM, Saltrick K, Mendicino RW, Catanzariti AR (2003) Peroneal tendon tears: a retrospective review. J Foot Ankle Surg 42(5):250-258

10. Roster B, Michelier P, Giza E (2015) Peroneal Tendon Disorders. Clin Sports Med 34(4):625-641

11. Saupe N, Mengiardi B, Pfirrmann CW, Vienne P, Seifert B, Zanetti M (2007) Anatomic variants associated with peroneal tendon disorders: MR imaging findings in volunteers with asymptomatic ankles. Radiology 242(2):509-517

12. Numkarunarunrote N, Malik A, Aguiar RO, Trudell DJ, Resnick D (2007) Retinacula of the foot and ankle: MRI with anatomic correlation in cadavers. AJR Am J Roentgenol 188(4):348-354
13. Ruiz JR, Christman RA, Hillstrom HJ (1993) Anatomical considerations of the peroneal tubercle. J Am Podiatr Med Assoc 83(10): $563-575$

14. Hyer CF, Dawson JM, Philbin TM, Berlet GC, Lee TH (2005) The peroneal tubercle: description, classification, and relevance to peroneus longus tendon pathology. Foot Ankle Int 26(11):947-950

15. Wang XT, Rosenberg ZS, Mechlin MB, Schweitzer ME (2005) Normal variants and diseases of the peroneal tendons and superior peroneal retinaculum: MR imaging features. Radiographics 25(3): 587-602

16. Sammarco GJ (1994) Peroneal tendon injuries. Orthop Clin North Am 25(1):135-145

17. Sobel M, Pavlov H, Geppert MJ, Thompson FM, DiCarlo EF, Davis WH (1994) Painful os peroneum syndrome: a spectrum of conditions responsible for plantar lateral foot pain. Foot Ankle Int 15(3):112-124

18. Mittal PS, Joshi SS, Chhaparwal R, Joshi SD (2014) Prevalence and Mophometry of Os Peroneum amongst Central Indians. J Clin Diagn Res 8(11):AC08-AC10

19. Bessette BJ, Hodge JC (1998) Diagnosis of the acute os peroneum fracture. Singapore Med J 39(7):326-327

20. Guimerá V, Lafuente A, Zambrana L, Rodriguez-Niedenführ M, Sañudo JR, Vazquez T (2015) The peroneocuboid joint: morphogenesis and anatomical study. J Anat 226(1):104-112

21. Martinoli C (2010) Musculoskeletal ultrasound: technical guidelines. Insights Imaging 1(3):99-141

22. Donovan A, Rosenberg ZS, Bencardino JT et al (2013) Plantar tendons of the foot: MR imaging and US. Radiographics 33(7): 2065-2085

23. Sofka CM, Adler RS, Saboeiro GR, Pavlov H (2010) Sonographic evaluation and sonographic-guided therapeutic options of lateral ankle pain: peroneal tendon pathology associated with the presence of an os peroneum. HSS J 6(2):177-181

24. Didolkar MM, Malone AL, Nunley JA 2nd, Dodd LG, Helms CA (2012) Pseudotear of the peroneus longus tendon on MRI, secondary to a fibrocartilaginous node. Skeletal Radiol 41(11):1419-1425

25. Jeppesen JB, Jensen FK, Falborg B, Madsen JL (2011) Bone scintigraphy in painful os peroneum syndrome. Clin Nucl Med 36(3): 209-211

26. Okazaki K, Nakashima S, Nomura S (2003) Stress fracture of an os peroneum. J Orthop Trauma 17(9):654-656

27. Crain MR, el-Khoury GY (1989) Stress fracture of the os peroneum. AJR Am J Roentgenol 152(2):430

28. Bashir WA, Lewis S, Cullen N, Connell DA (2009) Os peroneum friction syndrome complicated by sesamoid fatigue fracture: a new radiological diagnosis? Case report and literature review. Skeletal Radiol 38(2):181-186

29. O'Donnell P, Saifuddin A (2005) Cuboid oedema due to peroneus longus tendinopathy: a report of four cases. Skeletal Radiol 34(7): 381-388

30. Oh SJ, Kim YH, Kim SK, Kim MW (2012) Painful os peroneum syndrome presenting as lateral plantar foot pain. Ann Rehabil Med 36(1):163-166

31. Truong DT, Dussault RG, Kaplan PA (1995) Fracture of the os peroneum and rupture of the peroneus longus tendon as a complication of diabetic neuropathy. Skeletal Radiol 24(8): 626-628

32. Smania L, Craig JG, von Holsbeeck M (2007) Ultrasonographic findings in peroneus longus tendon rupture. J Ultrasound Med 26(2):243-246 
33. Brigido MK, Fessell DP, Jacobson JA et al (2005) Radiography and US of os peroneum fractures and associated peroneal tendon injuries: initial experience. Radiology 237(1):235-241

34. Bianchi S, Abdelwahab IF, Tegaldo G (1991) Fracture and posterior dislocation of the os peroneum associated with rupture of the peroneus longus tendon. Can Assoc Radiol J 42(5):340-344

35. Pessina R (1988) Os peroneum fracture. A case report. Clin Orthop Relat Res 227:261-264

36. Tehranzadeh J, Stoll DA, Gabriele OM (1984) Case report 271. Posterior migration of the os peroneum of the left foot, indicating a tear of the peroneal tendon. Skeletal Radiol 12(1):44- 47

37. Bianchi S, Delmi M, Molini L (2010) Ultrasound of peroneal tendons. Semin Musculoskelet Radiol 14(3):292-306
38. Maurer M, Lehrman J (2012) Significance of sesamoid ossification in peroneus longus tendon ruptures. J Foot Ankle Surg 51(3):352355

39. Peterson JJ, Bancroft LW (2001) Os peroneal fracture with associated peroneus longus tendinopathy. AJR Am J Roentgenol 177(1): $257-258$

40. Smith JT, Johnson AH, Heckman JD (2011) Nonoperative treatment of an os peroneum fracture in a high-level athlete: a case report. Clin Orthop Relat Res 469(5):1498-1501

41. Ho KK, Chan KB, Lui TH, Chow YY (2013) Tendoscopic-assisted repair of complete rupture of the peroneus longus associated with displaced fracture of the os peroneum-case report. Foot Ankle Int 34(11):1600-1604 